\title{
Multivariate statistical analysis and numerical modelling for the hydrogeological and hydrochemical characterization of a closed MSW landfill: the case study of Vizzolo-Predabissi.
}

\section{Analisi statistica multivariata e modellazione numerica per la caratterizzazione idrogeologica ed idrochimica di un ex-discarica di RSU: il caso di Vizzolo-Predabissi}

Letizia Fumagalli, Gennaro Alberto Stefania, Chiara Zanotti, Davide Sartirana, Giuseppe Raffaello Di Martino, Antonella Perosa, Piergiorgio Valentini, Marco Rotiroti, Tullia Bonomi

\begin{abstract}
Riassunto: Il presente lavoro ha l'obiettivo di valutare l'impatto sulle acque sotterranee della fuoriuscita di percolato verificatasi alla discarica di Vizzolo-Predabissi (Milano, Italia) durante la gestione post-chiusura. Sezioni idrogeologiche, ricostruzioni piezometriche, analisi spazio-temporale e multivariata dei dati idrochimici hanno consentito di definire il modello concettuale locale e di elaborare un modello di flusso, risolto e calibrato mediante i codici MODFLOW-NWT e PEST. I risultati dello studio hanno evidenziato come l'evento di fuoriuscita di percolato abbia determinato una contaminazione impulsiva attualmente in riduzione, che si differenzia come modalità e persistenza in zone con diverse caratteristiche ossido-riduttive
\end{abstract}

Abstract: This work aims at assessing the impact on groundwater resources of a leachate overflow occurred in the landfill of Vizzolo-Predabissi (Milan, Italy) after its closure. Hydrogeological sections, piezometric maps, spatio-temporal and multivariate analysis of hydrochemical data enabled to define a conceptual model and to build a groundwater flow model, solved and calibrated by means of MODFLOW-NWT and PEST. The results of the study pointed out that the leachate overflow produced an impulsive contamination, currently in attenuation, which behaved differently in areas with different redox characteristics.

Keywords: groundwater quality, contamination, leachate, calibration, cluster analysis

Parole chiave: qualità delle acque sotterranee, contaminazione, percolato, calibrazione, analisi dei cluster

\section{Chiara ZANOTTI 棒: \\ Letizia FUMAGALLI, Gennaro Alberto STEFANIA \\ Davide SARTIRANA, Marco ROTIROTI, Tullia BONOMI \\ Dipartimento di Scienze dell'Ambiente e della Terra, \\ Università degli Studi di Milano-Bicocca, Milano, Italia \\ c.zanotti@campus.unimib.it}

Giuseppe Raffaello DI MARTINO

Cem Ambiente S.p.a., Cavenago Brianza (MB), Italia

Antonella PEROSA, Piergiorgio VALENTINI

Città metropolitana di Milano, Area Ambiente, Settore Rifiuti e bonifiche,

Servizio Coordinamento Tecnico Rifiuti, Milano, Italia

Ricevuto/Received: 14 October 2019-Accettato/Accepted: 10 March 2020 Pubblicato online/Published online: 30 March 2020

This is an open access article under the CC BY-NC-ND license:

http://creativecommons.org/licenses/by-nc-nd/4.0/

(C) Associazione Acque Sotterranee 2020

\section{Introduzione}

Il problema dell'impatto delle discariche sulle acque sotterranee è di particolare rilievo in aree di pianura ad elevata urbanizzazione in cui un approvvigionamento idrico da acque sotterranee è spesso stato affiancato ad una tipologia di smaltimento dei rifiuti mediante utilizzo di ex-cave di sabbia e ghiaia. Per tale motivo in letteratura i casi di inquinamento da discarica sono attribuibili alla mancanza del contenimento o alla perdita della sua tenuta (Critto et al. 2003; Nigro et al. 2017; Preziosi et al. 2019; Stefania et al. 2018, 2019), mentre è anomalo un evento di inquinamento impulsivo generato dalla tracimazione di percolato, qui presentato, che sottolinea l'importanza di una corretta gestione post-chiusura.

Il presente lavoro tratta il caso della fuoriuscita di percolato verificatosi nella fase di post-chiusura della discarica di Vizzolo-Predabissi (Milano). Lobiettivo principale è quello di comprendere e valutare l'impatto che l'evento di tracimazione ha causato sulla qualità delle risorse idriche sotterranee in prossimità della discarica stessa. Gli studi sull'area sono stati promossi dalla Città Metropolitana di Milano, con risorse di R.L., con l'obiettivo di valutare la situazione corrente e la sua evoluzione.

\section{Materiali e Metodi \\ Area di Studio}

La discarica è ubicata nel comune di Vizzolo-Predabissi (Milano) su un terrazzo fluviale della valle del F.Lambro (Fig. 1). Il fiume delimita il terrazzo da Ovest a Sud, mentre a Nord corrono due linee ferroviarie ed a Ovest la Tangenziale TEEM. Impostata nel 1982 su di una vecchia discarica ed attiva fino al 2003, costruita secondo criteri di sicurezza, ha subito nel 2014 un evento di fuoriuscita di percolato che, infiltrandosi nel terreno, ha interessato le acque sotterranee. Responsabile dell'evento è stato il suo accumulo per mancata asportazione, incrementato dalle elevate precipitazioni dell'anno (1485.3 mm vs media di $895.3 \mathrm{~mm}$ ). Interventi di riduzione dei battenti del percolato hanno evitato successive fuoriuscite. La discarica è delimitata ad Ovest da una paratia che contiene un flusso idrico sospeso individuato alla base di un lotto di sopralzo della discarica. Sui lati Nord-Ovest e Nord-Est-Sud due dreni intercettano una falda sospesa irrigua, collettando il flusso al F. Lambro. Il fondo della discarica (76.7-80.9 m s.l.m.) non viene interessato da flussi idrici, 


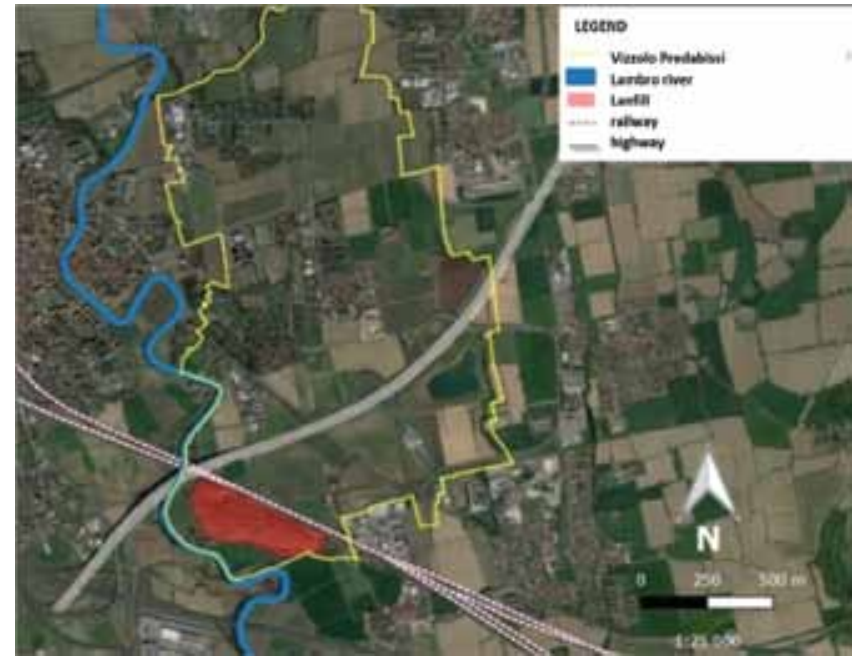

Fig. 1 - Ubicazione della discarica di Vizzolo-Predabissi, con F. Lambro, linee ferroviarie e superstrada TEEM.

Fig. 1 - Location of the Vizzolo-Predabissi landfill, with Lambro river, railways and TEEM highway.

essendo la sua quota superiore sia a quelle raggiunte dalla paratia (77- $69.5 \mathrm{~m}$ s.l.m.) e dai dreni (74.8-79.60 m s.l.m.), sia al livello idrometrico di piena eccezionale del F.Lambro (75.92 m s.l.m., Tempo di Ritorno 500 anni, Autorità del Bacino del Po, 2016).

\section{Modello concettuale idrogeologico}

Le n.8 sezioni idrogeologiche elaborate ad interpretazione dei dati stratigrafici hanno evidenziato la presenza di due sequenze sedimentarie (Fig. 2):

- $\quad$ sequenza superficiale limoso-argillosa (spessore $10-20 \mathrm{~m}$ ) di bassa permeabilità $\left(\mathrm{K}=10^{-9}-10^{-7} \mathrm{~m} / \mathrm{s}\right)$ che presenta nel settore centrale un corpo sabbioso $\mathrm{N}-\mathrm{S}$ sede di una falda libera arealmente discontinua;
- $\quad$ sequenza profonda sabbiosa (spessore 10-15 m) di buona conducibilità idraulica $\left(\mathrm{T}=1 \cdot 10^{-4}-4 \cdot 10^{-4} \mathrm{~m}^{2} / \mathrm{s}\right.$; Vercesi 2009) che è sede di una falda in pressione localmente connessa alla falda soprastante.

Gli andamenti temporali dei livelli piezometrici mensili suggeriscono la presenza di una ricarica irrigua estiva e di una meteorica autunnale, che determinano i massimi livelli piezometrici rispettivamente in settembre ed in novembre.

La mappa piezometrica della falda profonda del novembre 2017, anno con maggior numero di misure, evidenzia una direzione di flusso NE-SO, una diminuzione del gradiente idraulico sul terrazzo nella zona di contatto tra le falde e un aumento del gradiente verso il fiume, il cui drenaggio determina una morfologia piezometrica radiale divergente (Fig. 3).

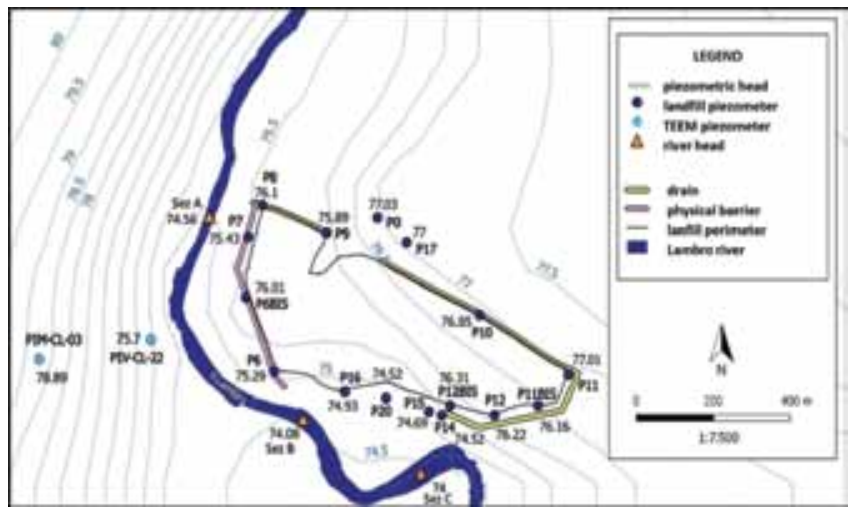

Fig. 3 - Piezometria della falda profonda (novembre 2017).

Fig. 3 - Piezometric map, deep aquifer (November 2017).

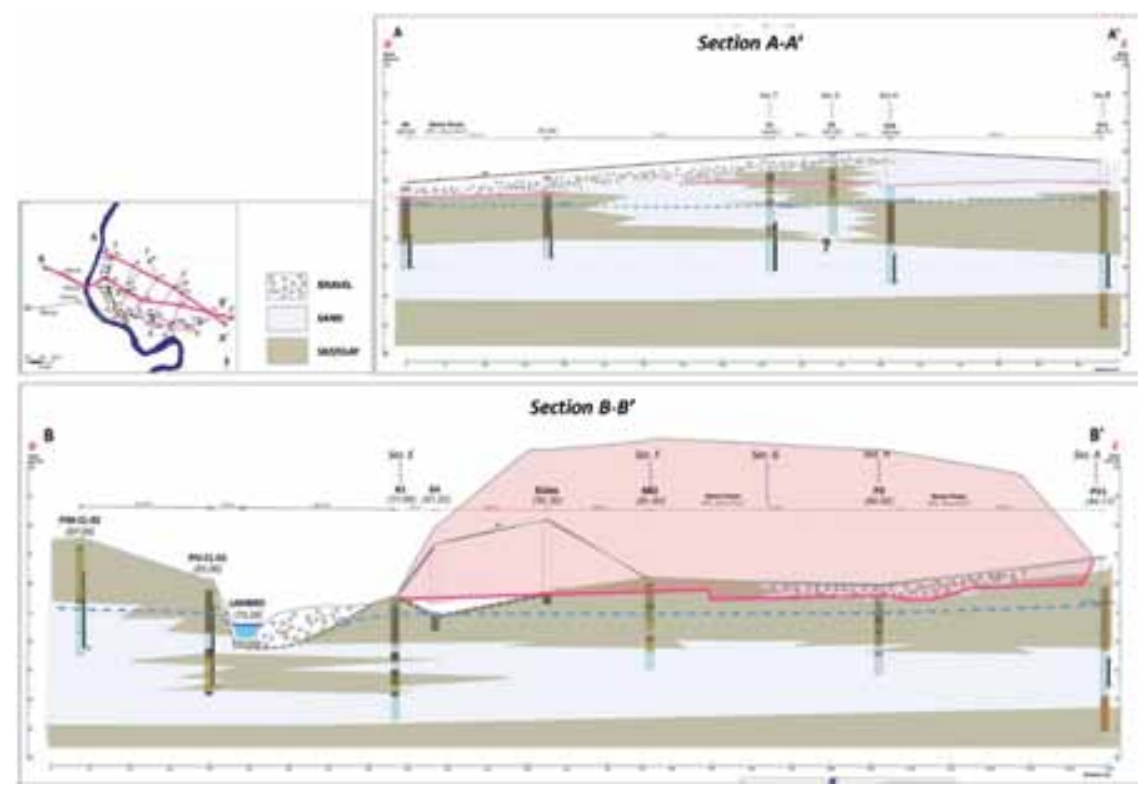

Fig. 2 - Sezioni idrogeologiche che mostrano le relazioni falda superficiale/falda profonda $\left(A-A^{\prime}\right)$ e l'ubicazione del corpo discarica (B-B').

Fig. 2 - Hydrogeological Sections showing the interaction between shallow and deep aquifers $\left(A-A^{\prime}\right)$ and the location of the landfill ( $\left.B-B^{\prime}\right)$. 


\section{Dati Utilizzati}

I dati sono relativi a 2 sondaggi, 21 piezometri discarica e 14 piezometri TEEM, per un totale di 38 stratigrafie. Le misure piezometriche mensili interessano il periodo marzo 2017-aprile 2018. I dati idrochimici sono 2396, acquisiti in campagne annuali di monitoraggio effettuate durante il funzionamento della discarica $(2000,2001,2002,2007$, 2008), in occasione della fuoriuscita di percolato (2014) e nella fase di gestione emergenziale $(2017,2018)$.

I parametri monitorati sono stati: $\mathrm{pH}$, Conducibilità Elettrica, Cloruri, Solfati, Ammonio, Nitrati, Calcio, Ferro, Manganese, Potassio, Arsenico, Rame, Cromo, Nichel, Piombo, Magnesio e Zinco.

I dati pre-2007 relativi a Ferro, Manganese e Arsenico risentono di differenti metodiche di campionamento/analisi (campioni non filtrati ed adozione di limiti di rilevabilità più alti), risultando pertanto non confrontabili con quelli delle campagne successive.

\section{Analisi statistica multivariata}

I dati idrochimici sono stati elaborati mediante cluster analysis (CA) al fine di identificare gruppi di dati tra loro simili per caratteristiche chimiche, con l'obiettivo di ottenere una zonazione dei piezometri che sia riassuntiva di tutti gli analiti considerati. I dati utilizzati nella CA sono relativi alle sole campagne 2014 e successive (2016 e 2017); questo perché la CA ha l'obiettivo principale di identificare se e come l'evento di tracimazione di percolato abbia avuto effetti diversi in zone diverse dell'area coinvolta. Per ciascun cluster identificato (ovvero gruppo di piezometri a caratteristiche/comportamento simile) è stata poi valutata l'evoluzione temporale di ciascun parametro.

La CA è stata elaborata a partire da una matrice di input costituita da 17 variabili (Conducibilità, $\mathrm{pH}$, Cloruri, Solfati, Ammonio, Nitrati, Calcio, Ferro, Manganese, Potassio, Arsenico, Rame, Cromo, Nichel, Piombo, Magnesio e Zinco) e 20 punti (i 20 piezometri attivi della discarica). I dati sono stati auto-scalati ed è stato utilizzato il metodo gerarchico di Ward (Ward 1963), basato sulla distanza euclidea al quadrato, come misura della similarità tra campioni. Ciascun piezometro è rappresentano dalla media delle concentrazioni relative ad ogni parametro, per il periodo considerato (20142017), in modo che singoli valori dei diversi parametri siano rappresentativi del comportamento chimico post evento di tracimazione. La scelta della media è subordinata all'obiettivo principale di questa CA, ovvero analizzare la variabilità spaziale degli effetti dell'evento impulsivo. La variabilità temporale è stata invece indagata attraverso grafici concentrazione/tempo per ciascun cluster.

\section{Modello di flusso}

Il modello di flusso, impostato sul modello concettuale idrogeologico, è stato implementato utilizzando il codice MODFLOW-NWT (Niswonger et al. 2011) con interfaccia GWV.

Il modello è stato calibrato secondo un approccio inverso utilizzando il codice PEST (Parameters ESTimation code; Doherty 2004).

\section{Impostazione del Modello di Flusso}

Il modello presenta celle di $5 \times 5 \mathrm{~m}$ su una griglia di calcolo di 400 righe, 300 colonne e 6 strati (Fig. 4) che rappresentano:

- Strato 1: gli elementi superficiali, quali discarica e alveo fluviale (spessore 2-25 m);

- Strato 2: il fondo della discarica impermeabilizzato (spessore $1.5 \mathrm{~m}$ );

- Strato 3, 4, 5: l'acquifero superficiale eterogeneo (spessore $3 \mathrm{~m} \mathrm{x} 3$ );

- Strato 6: l'acquifero profondo omogeneo (spessore di $10 \mathrm{~m}$ ).

La simulazione, in regime stazionario, è relativa al mese di novembre 2017.

Le condizioni imposte al contorno riproducono la configurazione idrogeologica dell'area mediante:

- Carico Costante (strati 1-6): carico idraulico della falda di $76.80 \mathrm{~m}$ s.l.m. al limite ad ovest (sinistra Lambro) e di 80.5 m s.l.m. al limite a NE (destra Lambro);

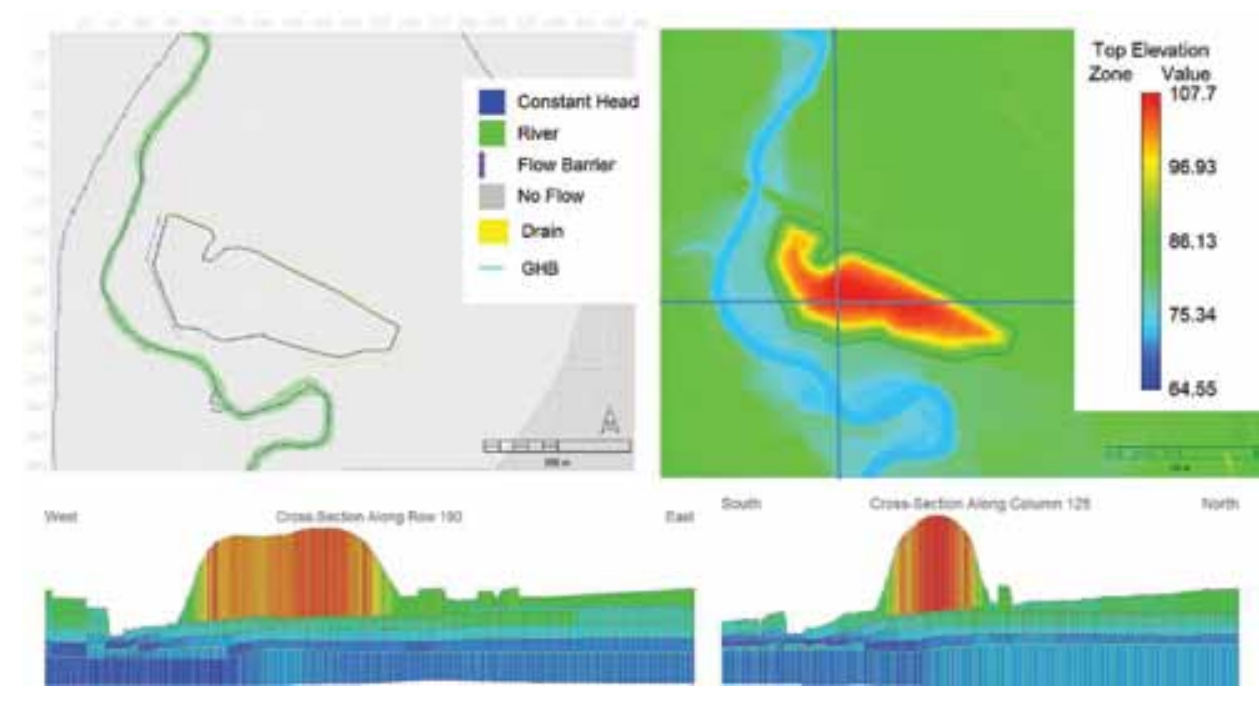

Fig. 4 - Griglia di calcolo del modello e sezione trasversale (riga n.140) con la quota del tetto dello strato in colore.

Fig. 4 - Model grid and classification by colors of the height of the layers top (cross section, row n.140). 
- $\quad$ Flusso Nullo (strati 1-6): ad indicare la direzione di flusso regionale nel settore SE del modello privo di dati;

- Flusso Dipendente dal Carico (RIVER) (strato 1): fiume, quota del letto (71.9-70.8 m s.l.m.); larghezza (30 m), quota del pelo libero, (73.42-72.91 m s.l.m.); conducibilità del letto $\left(1.10^{-5} \mathrm{~m} / \mathrm{s}\right)$;

- $\quad$ Flusso Dipendente dal Carico (DRN) (strato 3): dreno, base 79.6-74.8 m s.l.m., pendenza 0.24-1.06\%, conducibilità idraulica $\left(1 \cdot 10^{-3} \mathrm{~m} / \mathrm{s}\right)$;

- $\quad$ Flusso Dipendente dal Carico (GHB) (strato 4): allontana le condizioni di carico della falda per evitare che il flusso nullo del limite Sud del modello controlli l'andamento piezometrico

- Barriera al Flusso Orizzontale (WALL) (strati 1-4): paratia, spessore di $0.4 \mathrm{~m}$, conducibilità $1 \cdot 10^{-11} \mathrm{~m} / \mathrm{s}$.

- $\quad$ Flusso Costante $(\mathrm{RCH})$ : ricarica della falda, stimata in 0.0002 $\mathrm{m} / \mathrm{d}$ nell'area e in $0.00001 \mathrm{~m} / \mathrm{d}$ nella zona della discarica (12\% e $1 \%$ della precipitazione media).

Per quanto concerne la conducibilità idraulica, le tessiture relative alle 38 stratigrafie sono state tradotte, a scala metrica, in valori di conducibilità mediante la banca dati TANGRAM (Bonomi et al. 2014); la media geometrica dei valori compresi in ognuno dei 6 strati, per ogni stratigrafia, costituisce una griglia di 185 valori, compresi tra $1.00 \cdot 10^{-7} \mathrm{~m} / \mathrm{s}$ e $6.67 \cdot 10^{-4} \mathrm{~m} / \mathrm{s}$, utilizzati come valori iniziali per i "pilot points" utilizzati nella fase di calibratura per ottenere la distribuzione della conducibilità nel dominio del modello.

\section{Calibrazione e analisi di sensitività}

La calibrazione mediante PEST ha impiegato la tecnica SVD-assist accoppiata all'uso di "pilot-points", permettendo da un lato di inglobare nel processo le informazioni disponibili sulla conducibilità idraulica desunti dalle stratigrafie (mediante regolarizzazione di Tikhonov) e dall'altro ridurre il tempo di calcolo concentrandolo sulle combinazioni di parametri maggiormente sensibili al processo (metodo dei sottospazi) (Doherty 2004, 2010a, 2010b).

In particolare, sono state utilizzate due griglie di pilotpoints disposti nei 6 strati:

- una griglia di 185 valori stimati da stratigrafia tramite TANGRAM, cui è stato assegnato un intervallo di variazione di $\pm 25 \%$ del proprio valore;

- una griglia ausiliaria di 888 valori (125 m di lato) pari alla media geometrica dei valori estremi stimati da stratigrafia, con intervallo di variazione assegnato tra $7.5 \cdot 10^{-8}-8.34 \cdot 10^{-4} \mathrm{~m} / \mathrm{s}( \pm 25 \%$ dei valori estremi).

Oltre alla conducibilità idraulica del sottosuolo, il processo di calibrazione ha riguardato anche la conducibilità idraulica del letto del fiume ed il carico costante della falda a NE; in tale fase, alla prima è stato assegnato un intervallo di variazione tra $1 \cdot 10^{-7}$ e $1 \cdot 10^{-3} \mathrm{~m} / \mathrm{s}$ (valore iniziale $1.10^{-5} \mathrm{~m} / \mathrm{s}$ ), alla seconda un intervallo di $\pm 4 \mathrm{~m}$ s.l.m (valore iniziale $80.5 \mathrm{~m} \mathrm{s.l.m.).}$

In considerazione dell'incertezza dei valori attribuiti ai parametri ricarica $(0.00001 \mathrm{~m} / \mathrm{d}$ e 0.0002 m/d) e conducibilità del dreno $(0.001 \mathrm{~m} / \mathrm{s})$, questi sono stati sottoposti ad una analisi di sensitività assegnando 7 moltiplicatori $(0.25,0.5$,
$0.75,1,1.25,1.5,1.75)$ al valore iniziale ed analizzando le somme quadratiche dei residui ottenute nelle 7 simulazioni effettuate.

\section{Simulazione mediante MODPATH}

Con l'obiettivo di ricostruire il percorso seguito dal contaminante in seguito all'evento, è stato utilizzata la tecnica del "tracciamento di particelle" sul modello calibrato, con la quale il codice MODPATH (Pollock 2016) visualizza il flusso advettivo (conservativo).

A tal fine delle particelle, 1 per cella, sono state inserite nel modello in una fascia intorno alla discarica, in modo da simulare quattro diversi scenari di profondità di infiltrazione di percolato, tra 6 e $20 \mathrm{~m}$, ovvero alle basi degli strati 2, 3, 4 e 5 .

\section{Risultati \\ Cluster Analysis}

I risultati della $\mathrm{CA}$, rappresentati dal dendrogramma di Figura 5, hanno portato all'individuazione di 8 clusters. Dalla mappa con l'ubicazione dei piezometri appartenenti a ciascun cluster (Fig. 6) e dai grafici box plots (Turkey 1977) relativi ai valori misurati tra il 2014 e il 2017 nei piezometri suddivisi per cluster (Fig. 7), è possibile evidenziare che:

- il cluster 1, con i piezometri P12bis, P15, P1, P14, P6, P19, P20, P10, P0 e P5, ubicati nella parte centrale della discarica, presenta valori inferiori a quelli degli altri cluster per quanto riguarda tutte le variabili considerate;

- il cluster 2, con i piezometri P11, P11bis, P12, ubicati ad est, si caratterizza per elevati valori di Ferro, Arsenico e Manganese, inferiori solo a quelli del cluster 3 (P13) che rappresenta i massimi valori del dataset considerato;

- $\quad$ i clusters 3 (P13), 4 (P13bis), 5 (P20bis), 6 (P6bis) e 7 (P7) sono "singleton", ovvero cluster composti da un singolo elemento, e fanno riferimento a piezometri che si caratterizzano per valori anomali di alcuni parametri rispetto ai restanti piezometri;

- $\quad$ il cluster 8, con i piezometri P8 e P9, ubicati a nord-ovest, si caratterizza per elevati valori di Ferro e Manganese, mentre l'Arsenico rimane su valori inferiori a quelli del cluster 2 .

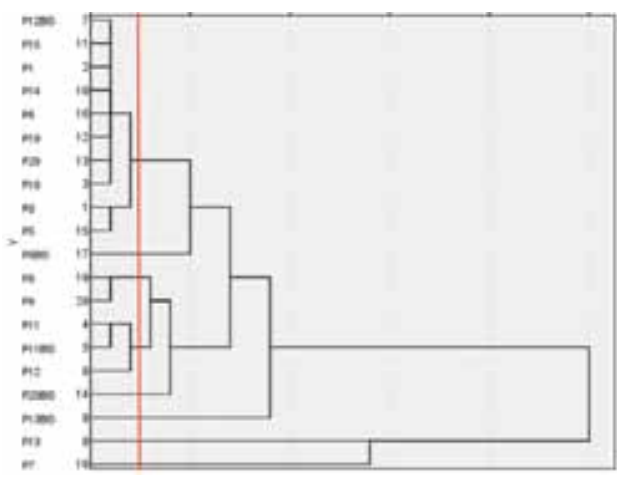

Fig. 5 - Dendrogramma gerarchico utilizzato per l'individuazione degli 8 clusters

Fig. 5 - Dendrogram and hierarchical clustering to produce the 8 Clusters. 


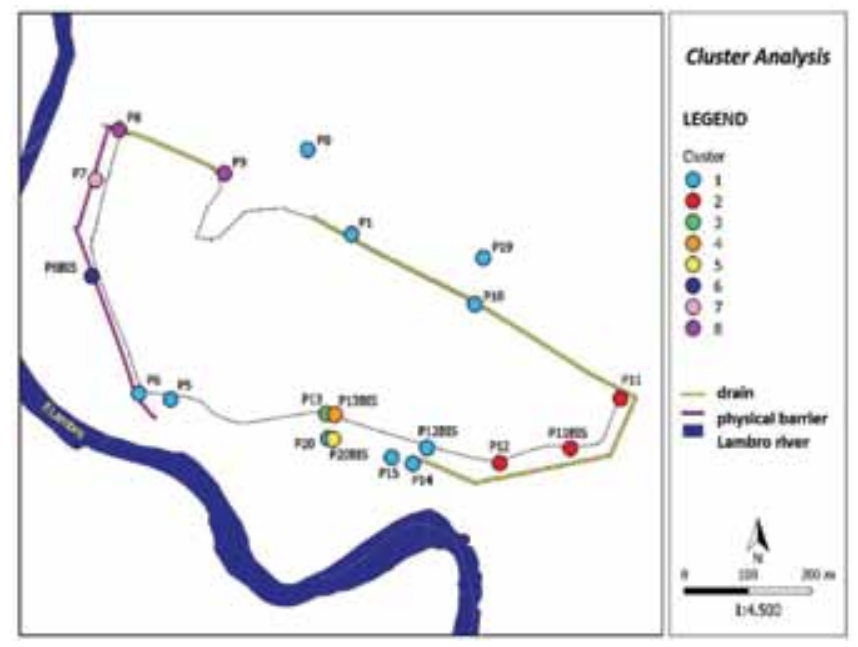

Fig. 6 - Mappa dei piezometri classificati in accordo con i risultati della cluster analysis.

Fig. 6 - Piezometers locations and their classification into cluster analysis classification.
Levoluzione temporale delle concentrazioni è riportata in Figura 8, a titolo di esempio solo per i parametri Conducibilità Elettrica e Arsenico. Per quanto riguarda la conducibilità, è chiaramente visibile come tutti i piezometri abbiano risentito dell'evento del 2014, che ha determinato un innalzamento generale dei valori. Negli anni successivi però i valori dei cluster 1, 2, 5, 6 e 8 tornano ad assestarsi su valori prossimi a quelli antecedenti al 2014, mentre solo il cluster 7 registra valori ancora alti nel 2017. Per quanto riguarda l'Arsenico, gli aumenti di concentrazione collegabili all'evento del 2014, dove registrati, sono rientrati negli anni successivi ad eccezione dei cluster 2 e 3 (P13), dove concentrazioni elevate sono state misurate anche per l'anno 2017. In particolare, è da segnalare il trend del P11bis (cluster 2) che ha avuto un progressivo aumento di concentrazione dal 2014 al 2017.

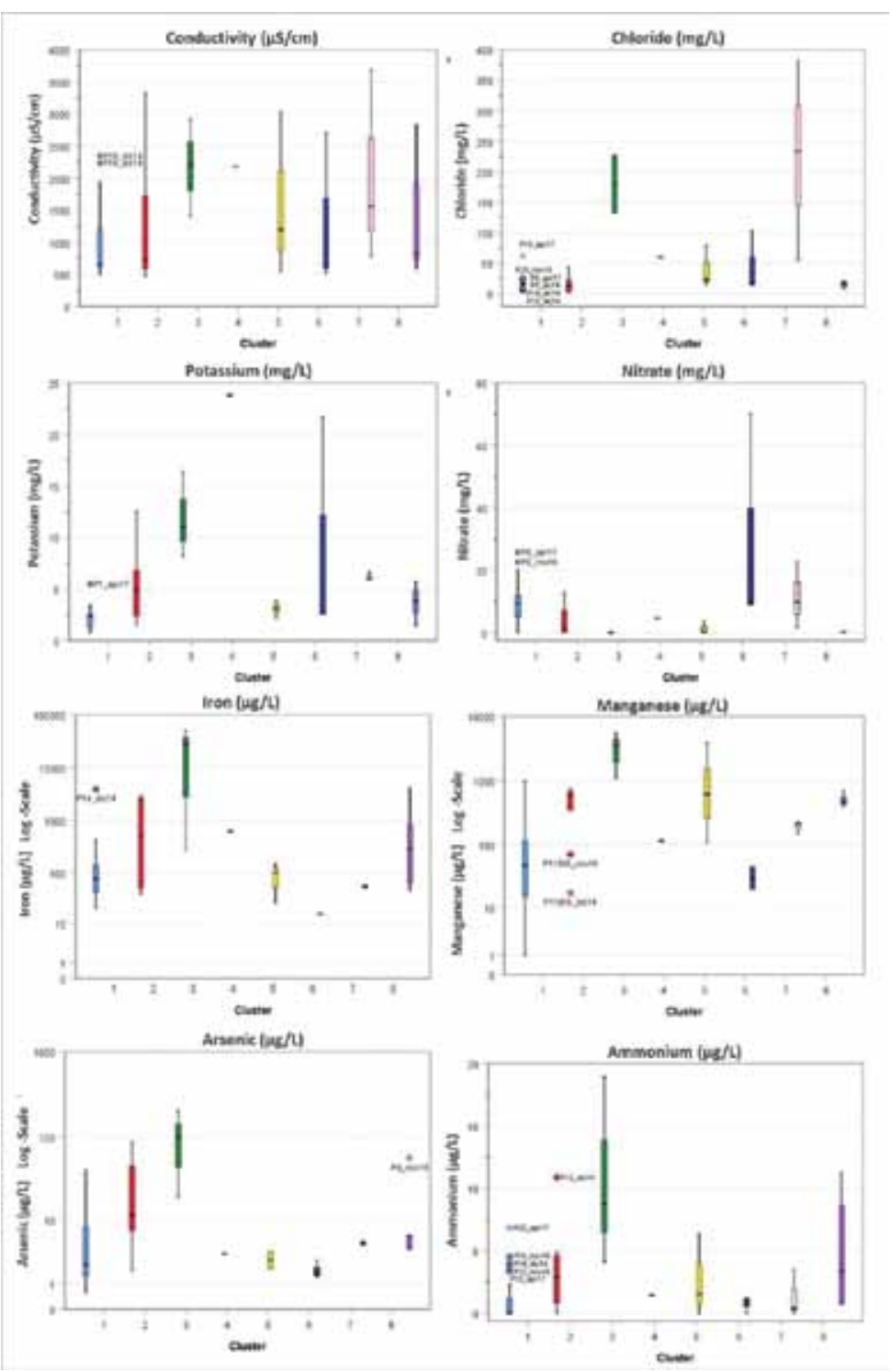

Fig. 7 - Mappa dei piezometri classificati in accordo con i risultati della cluster analysis

Fig. 7 - Piezometers locations and their classification into cluster analysis classification. 


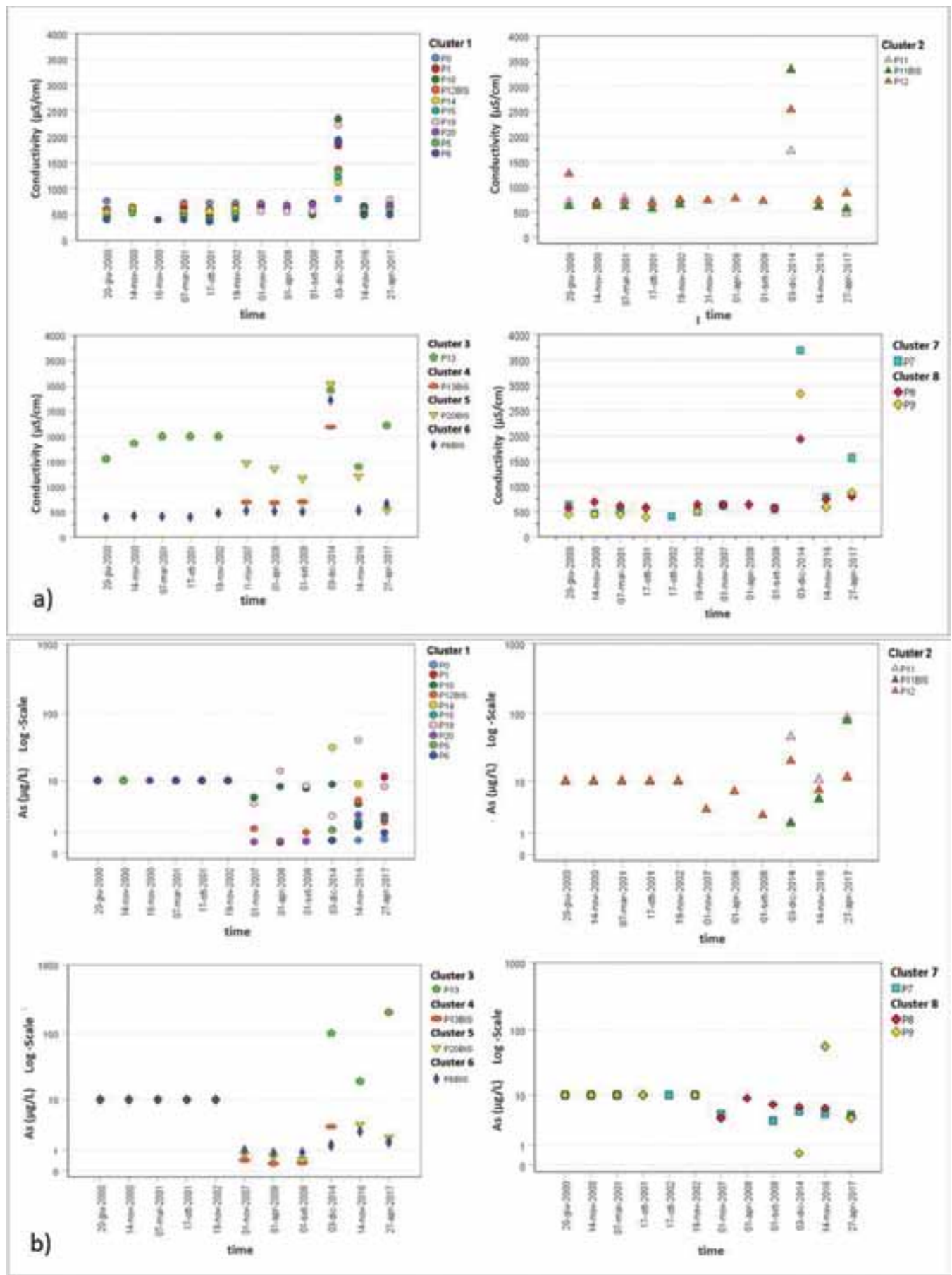

Fig. 8 - Evoluzione temporale dei valori di Conducibilità Elettrica (a) e Arsenico (b) per i piezometri raggruppati in clusters.

Fig. 8 - Temporal evolution of measured values of Electric Conductivity (a) and Arsenic (b) for piezometers grouped in clusters.

\section{Modello di Flusso e Tracciamento delle Particelle}

La tecnica di calibrazione adottata (tramite codice PEST ed utilizzo dei "pilot points") ha ottenuto una ricostruzione spaziale della conducibilità idraulica nel dominio del modello con valori compresi tra $1.16 \cdot 10^{-9}-1.2 \cdot 10^{-3} \mathrm{~m} / \mathrm{s}$. La Figura 9 illustra la distribuzione di tali valori negli strati del modello, con presenza di zone a bassa conducibilità nei settori est ed ovest dell'area discarica non presenti nel sesto strato omogeneamente sabbioso. Nello strato 2 appare l'impermeabilizzazione della base della discarica.
Relativamente alla calibrazione della conducibilità idraulica del letto del fiume e del limite a carico costante a NE dell'area, i valori risultanti sono stati rispettivamente $1.77 \cdot 10^{-6} \mathrm{~m} / \mathrm{s}$ e 82.5 m s.l.m.

Per quanto riguarda all'analisi di sensibilità della ricarica e della conducibilità del dreno essa conferma il valore della ricarica areale $(0.0002 \mathrm{~m} / \mathrm{d})$ mentre indica l'insensibilità del modello a variazioni del valore di ricarica nell'area discarica $(0.00001 \mathrm{~m} / \mathrm{d})$ e della conducibilità del dreno $(0.001 \mathrm{~m} / \mathrm{s})$, valori che sono quindi stati confermati. 

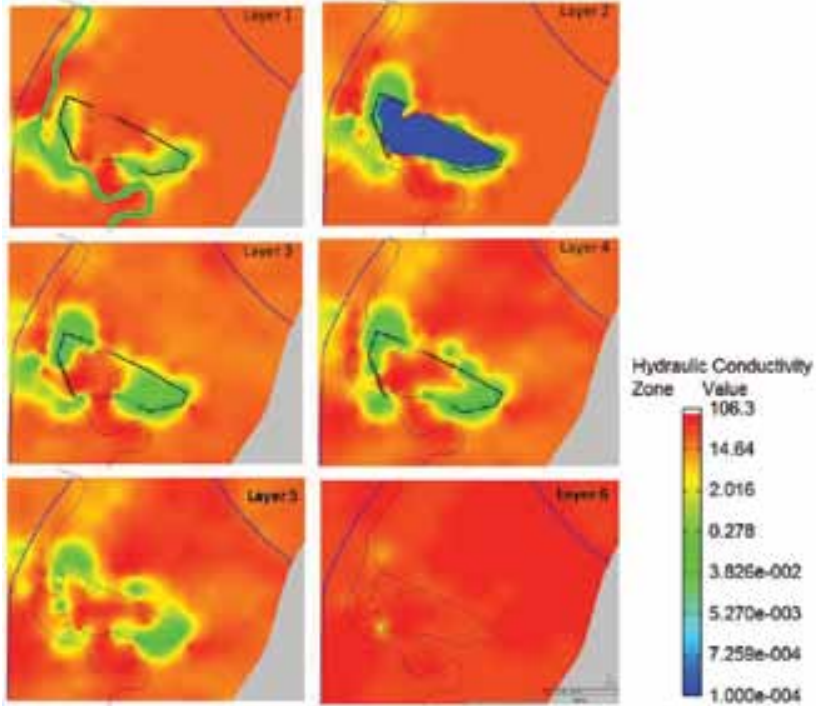

1.0000004

Fig. 9 - Distribuzione spaziale della conducibilità idraulica ottenuta a seguito del processo di calibrazione.

Fig. 9 - Spatial distribution of the hydraulic conductivity obtained following the calibration process.
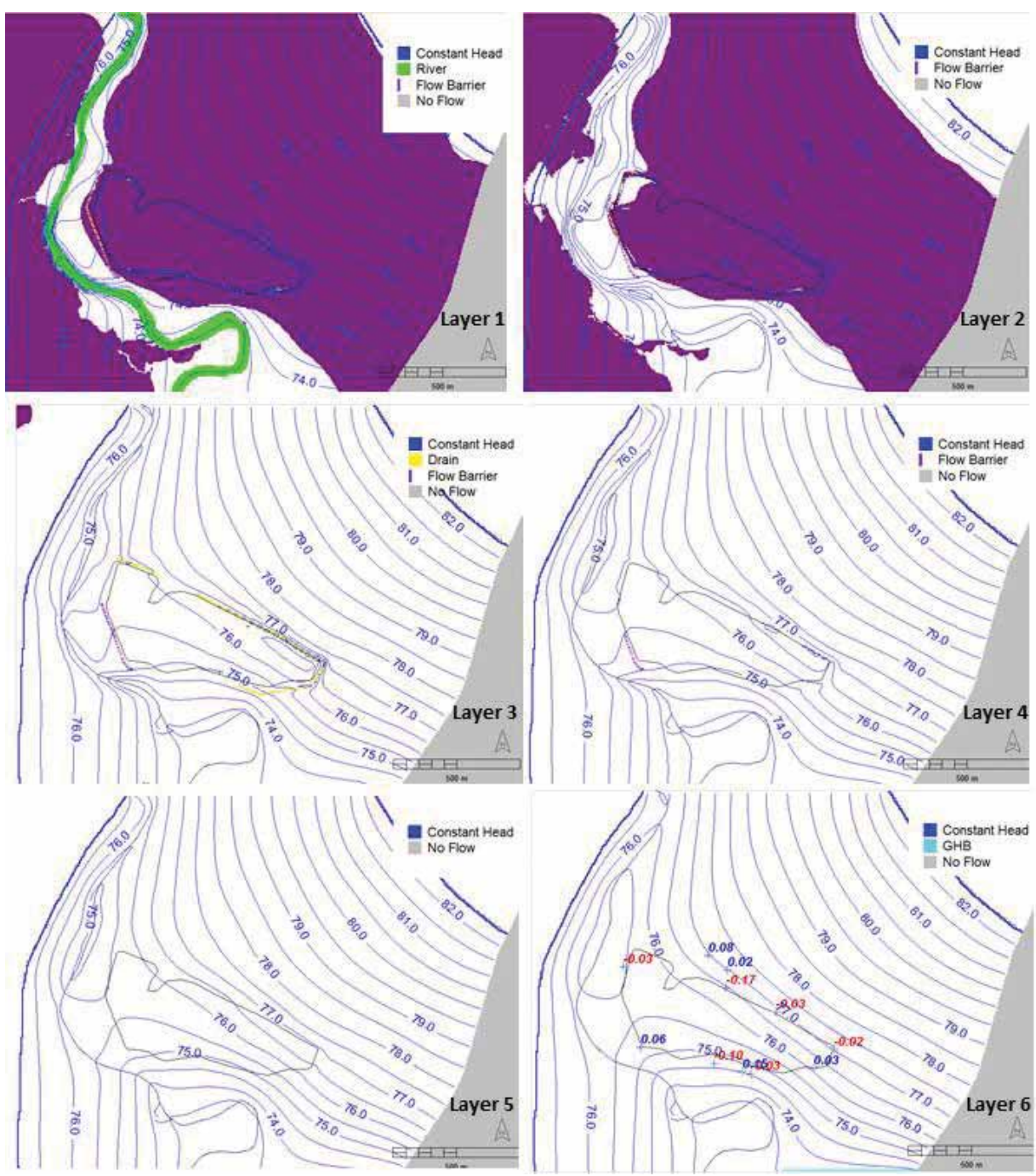

Il modello calibrato presenta una piezometria della falda (Figura 10) in cui i residui rispetto alle misure sono compresi tra -0.171 e $+0.15 \mathrm{~m}$ (media $-0.003 \mathrm{~m}$ ), mentre la radice dell'errore quadratico medio normalizzato (nRMSE) è 0.033 (Tabella 1, Figura 11).

Tab. 1 - Descrittori statistici di sintesi della soluzione calibrata.

Tab. 1 - Summary statistical descriptors of the calibrated solution.

\begin{tabular}{|l|l|}
\hline Residual Mean (m) & -0.003 \\
\hline RSS (m) & 0.077 \\
\hline Minimum Residual (m) & -0.171 \\
\hline Maximum Residual (m) & 0.15 \\
\hline Num.Observation (ad.) & 11 \\
\hline Hmax-Hmin (m) & 2.51 \\
\hline nRMSE (ad.) & 0.033 \\
\hline
\end{tabular}

Fig. 10 - Piezometrie simulate nei singoli strati (viola: celle asciutte). Nello strato 6 i residui dei target: sovrastima (rosso), sottostima (blu).

Fig. 10 - Simulated piezometric maps of each layer (purple: dry cells). Residual values of the targets in layer 6: overestimation (red), underestimation (blue). 


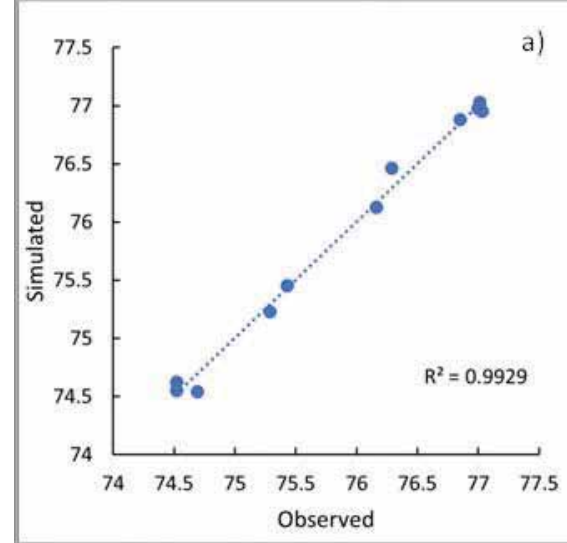

In termini di bilancio (Figura 12), il modello riceve $212 \mathrm{~L} / \mathrm{s}$ dal flusso di falda al limite NE e $6 \mathrm{~L} / \mathrm{s}$ per ricarica, mentre ne perde $26.6 \mathrm{~L} / \mathrm{s}$ per flusso di falda dal limite $\mathrm{S}$ e, per drenaggio del fiume e del dreno, rispettivamente 189 e $4 \mathrm{~L} / \mathrm{s}(0.013 \mathrm{~L} / \mathrm{s}$ e $0.049 \mathrm{~L} / \mathrm{s}$ per singola cella). L'errore del bilancio di massa è 8.3.10-6\%

Il tracciamento delle particelle (Figura 13) evidenzia i percorsi del flusso advettivo tra diversi strati, con diverse colorazioni in funzione dello strato attraversato.

Le particelle, in funzione della profondità alla quale si originano, interessano la zona centrale, sede della falda superficiale, con direzione verso Sud, e la zona Nord-Ovest, drenate dal F. Lambro (origine: base strati 2, 3, 4); più in profondità (origine: base strato 5) si approfondiscono nella falda semiconfinata (strato 6) per emergere a Sud drenate dal fiume. b)

Fig. 11 - Confronto dei valori osservati (m s.l.m.) vs simulati (m s.l.m.) (a) e dei valori osservati (m s.l.m.) vs residui $(\mathrm{m})(\mathrm{b})$.

Fig. 11 - Comparison of observed ( $m$ a.s.l.) vs simulated ( $m$ a.s.l.) values (a) and observed values ( $m$ a.s.l.) vs residuals $(m)(b)$

Observed

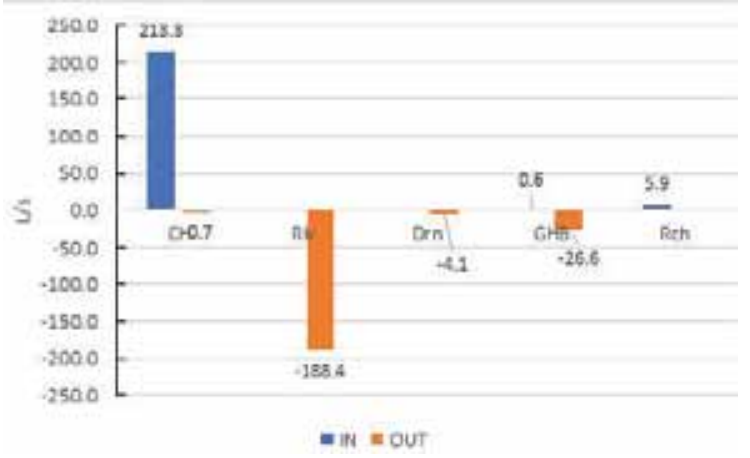

Fig. 12 - Bilancio di massa del modello con entrate (blu) e uscite (arancio) rispetto a Limite a Carico Costante (CH), Fiume (Riv), Dreno (Drn), Limite a Carico Generale (GHB), Ricarica (Rch).

Fig. 12 - Model mass balance with inflow (blue) and outflow (orange) with respect to: Constant Head (CH), River (Riv), Drain (Drn), General Head Boundary (GHB), Recharge (Rch).

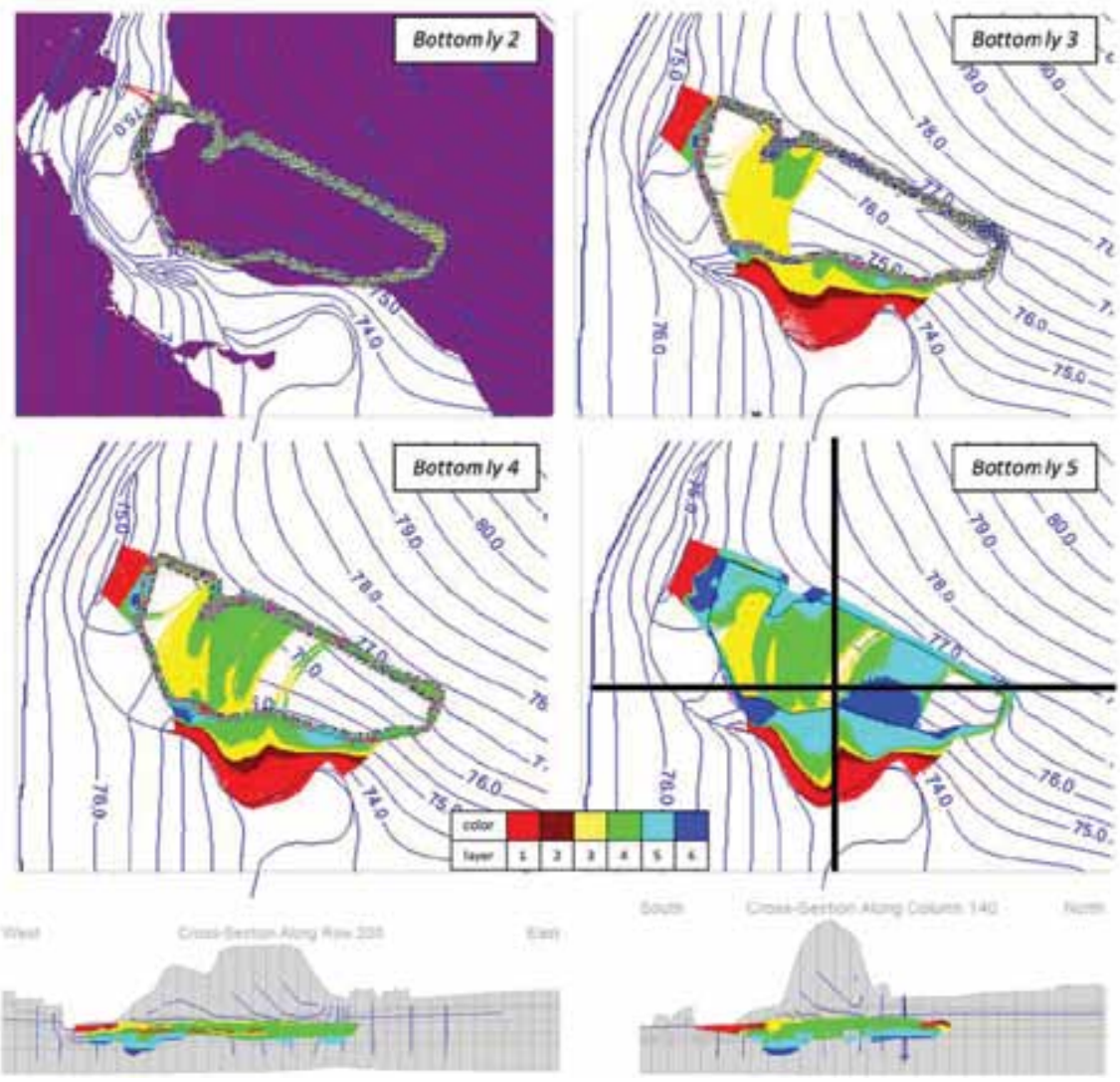

Fig. 13 - Confronto dei valori osservati (m s.l.m.) vs simulati (m s.l.m.) (a) e dei valori osservati (m s.l.m.) vs residui (m) (b)

Fig. 13 - Comparison of observed ( $m$ a.s.l.) vs simulated ( $m$ a.s.l.) values $(a)$ and observed values ( $m$ a.s.l.) vs residuals $(m)(b)$. 
Si osserva che il fiume è il ricettore ultimo dei flussi nell'area discarica.

\section{Discussione}

Landamento temporale dei dati chimici (Fig. 8) ha evidenziato l'origine impulsiva della contaminazione e il coinvolgimento di entrambe le falde, essendo la falda profonda non confinata nel settore centrale ed in comunicazione con quella superficiale.

Gli 8 clusters ottenuti dalla CA, analizzati insieme alle caratteristiche idrogeologiche ed ai risultati modellistici, forniscono una spiegazione esauriente dei fenomeni in corso nell'area in base all'individuazione di zone con caratteristiche peculiari:

- il cluster 1, che raggruppa 10 piezometri della zona centrale della discarica, presenta caratteristiche chimiche ossidate. Questo è dovuto al fatto che in questa zona i livelli limosi ed argillosi sono meno abbondanti, permettendo così una maggiore ricarica da parte di acque ben ossigenate. In questa zona l'evento del 2014 ha portato ad un aumento principalmente di conducibilità elettrica, che poi è rientrato completamente nell'anno 2016. Quindi quest'area ha dimostrato una buona capacità di attenuazione naturale dell'evento del 2014;

- il cluster 2, che raggruppa i 3 piezometri nella zona orientale della discarica, presenta caratteristiche chimiche ridotte. Questo è collegabile alla presenza di consistenti livelli argillosi e limosi in questa zona, che non permettono una facile ricarica da parte di acque ossigenate. In questi piezometri l'evento del 2014 ha generato un aumento nelle concentrazioni di $\mathrm{Mn}, \mathrm{Fe}$ ed As. Questo è collegabile alla presenza di conduzioni riducenti, infatti, in assenza di ossigeno, la sostanza organica (contenuta nel percolato) viene degradata tramite i processi di dissoluzione riduttiva degli ossidi di ferro e manganese contenuti nell'acquifero, portando ad alte concentrazioni di ferro e manganese nella falda. Se questi processi si spingono ad un grado avanzato, portano anche ad un aumento delle concentrazioni di As, che si trova generalmente adsorbito sugli ossidi di ferro e manganese (Rotiroti et al. 2014). Le concentrazioni di As in crescita riscontrate nell'anno 2017 in questi piezometri (in particolare nel P11 e P11bis) potrebbero essere ancora legate all'evento del 2014;

- il cluster 8, formato dai 2 piezometri della zona nordoccidentale della discarica, presenta caratteristiche chimiche ridotte. Come per il cluster 2 , questo è collegabile alla presenza di consistenti livelli argillosi e limosi in questa zona, che non permettono una facile ricarica da parte di acque ossigenate. In questi piezometri l'evento del 2014 ha generato un aumento nelle concentrazioni di $\mathrm{Mn}$ e $\mathrm{Fe}$, in maniera analoga a quanto descritto per il cluster 2. A differenza del cluster 2 però, non si sono registrati aumenti significativi di As, ad eccezione del P9 per il solo anno 2016. Questo può essere dovuto al fatto che in questa zona si sono instaurate condizioni riducenti meno avanzate rispetto a quelle del cluster 2 , o comunque le condizioni riducenti avanzate che si sono instaurate (nel P9 per il 2016) sono poi rientrate in tempi più brevi (basse concentrazioni di As nel P9 nel 2017);

- $\quad$ i clusters 3-7 esprimono condizioni di impatto particolare ; da sottolineare i singoli piezometri ad ovest fortemente influenzati dall'evento del 2014 che ha generato elevatissimi valori di Cloruri, oltre ad alti valori di Conducibilità, Potassio e Nitrati (cluster 6) e di Nichel (cluster 7).

Le caratteristiche idrogeologiche descritte dalle sezioni appaiono ben riprodotte nel modello grazie sia alla capacità di TANGRAM di derivare valori di conducibilità dalle tessiture, sia alla potenzialità di PEST di calibrare tali valori e interpolarli spazialmente mediante pilot-points.

Il flusso idrico modellato denuncia la zona di contatto delle due falde, con una riduzione del gradiente idrico, e l'azione drenante del fiume, presso il quale le celle risultano sature.

Il percorso delle linee di flusso tracciate da MODPATH indica che, con l'approfondirsi della infiltrazione del percolato (particelle) viene dapprima interessata l'intera falda superficiale arealmente poco estesa (strati 3-4) e, più in profondità (strato 5) il tragitto di particelle posizionale più in profondità interessa anche la falda profonda (strato 6) suggerendo per essa un pennacchio più ampio. Relativamente al dreno di presidio alla discarica, esso intercetta le particelle, quindi i flussi dalla discarica, convogliandoli al fiume. Da ultimo il F.Lambro si dimostra essere stato il recettore ultimo della contaminazione, in quanto ogni particella con origine nell'area discarica raggiunge il fiume.

\section{Conclusioni}

Un evento di tracimazione di percolato dalla discarica di Vizzolo Predabissi verificatosi nel 2014 è stato studiato dal punto di vista idrogeologico con l'ausilio di modelli numerici di flusso delle acque sotterranee ed analisi statistica multivariata dei dati chimici.

La struttura idrogeologica del terrazzo fluviale su cui è posta la discarica presenta una falda profonda sabbiosa confinata da orizzonti a bassa permeabilità, interrotti nel settore centrale da un corpo sabbioso sede di una falda superficiale di limitata estensione, in contatto con la sottostante.

Dal punto di vista chimico i risultati delle elaborazioni svolte indicano l'impulsività della contaminazione legata all'evento, che ha interessato entrambe le falde, e le differenze nelle modalità di contaminazione e nella sua persistenza in diverse zone con differenti caratteristiche redox legate a peculiari caratteristiche idrogeologiche.

Nella zona centrale la contaminazione, visibile per un aumento di conducibilità, si è velocemente risolta grazie al contatto tra le falde che determina condizioni ossidate anche in profondità e alta trasmissività. Essa ha creato invece nelle zone nord-occidentale ed orientale, dove la falda è confinata e sono presenti condizioni riducenti più o meno avanzate, un aumento di Ferro e Manganese, rientrato negli anni successivi, e localmente anche Arsenico, più persistente. 
Ad ovest singoli piezometri indicano un forte impatto dell'evento con valori di Cloruri molto elevati, Potassio, Nitrati e Nichel, in quanto direttamente interessati dai flussi verso ovest per drenaggio del fiume.

Il modello di flusso ha evidenziato i buoni risultati ottenuti dall'uso integrato della banca dati TANGRAM per la stima di valori iniziali di conducibilità idraulica e di PEST per la calibrazione degli stessi mediante pilot-points. I risultati del modello hanno completato lo scenario descritto, sottolineando la funzionalità del dreno, capace di intercettare anche flussi dalla discarica, e l'azione drenante del Lambro, che ha rappresentato il recettore finale della contaminazione verificatasi nell'area della discarica.

Significative sono state le indicazioni tratte dalla simulazione del percorso di particelle imposte al contorno della discarica che simulassero il destino del percolato infiltratosi a diverse profondità: ne risulta che, a limitate profondità, una contaminazione viene veicolata nella limitata falda superficiale verso sud, o da quella profonda ad ovest verso il fiume, a maggiore profondità si interessa la falda confinata, approfondendosi ed emergendo nel fiume a sud.

Lo studio ha messo in evidenza che l'utilizzo di diversi approcci (idrogeologico, idrochimico, statistico e modellistico) per l'analisi dell'evento e dei relativi effetti è complementare nel raggiungimento di risultati, integrandosi tra loro in una migliore comprensione dei fenomeni indagati con quindi maggiori possibilità di intervenire nella protezione delle acque sotterranee.

Nel caso presente prescrizioni di controllo e prevenzione hanno compreso il miglioramento della rete di monitoraggio al fine di controllare $i$ flussi in arrivo ed in uscita in punti strategici.

Ringraziamenti: Il presente lavoro è stato finanziato da CEM Ambiente S.p.a. tramite convenzione scientifica n. 2017-CONV250044 con l'Università di Milano-Bicocca.

\section{BIBLIOGRAFIA}

Autorità di Bacino del Fiume Po (2016). Piano per la valutazione e la gestione del rischio di alluvioni, Art. 7 della Direttiva 2007/60/CE e del D.lgs. n. 49 del 23.02.2010

Bonomi T., Fumagali L., Rotiroti M., Bellani A., Cavallin A. (2014) The hydrogeological well database TANGRAM: a tool for data processing to support groundwater assessment. Acque Sotterranee - Italian Journal of Groundwater 3(2): 35-45, doi:10.7343/AS072-14-0098

Critto A., Carlon C., Marcomini A. (2003) Characterization of contaminated soil and groundwater surrounding an illegal landfill (S. Giuliano, Venice, Italy) by principal component analysis and kriging. Environmental Pollution 122(2): 235-244. doi:10.1016/ S0269-7491(02)00296-8

Doherty J. (2004) PEST Model-Independent Parameter Estimation. User Manual (5th ed). Corinda, Australia: Watermark Numerical Computing.

Doherty J. (2010a). PEST Model Independent Parameter Estimation User Manual: 5th Edition. Watermark Numerical Computing. http://www.pesthomepage.org/Downloads.php

Doherty J. (2010b). Addendum to the PEST manual. Watermark Numerical Computing. http://www.pesthomepage.org/Downloads.php

Nigro A., Sappa G., Barbieri M. (2017) Application of boron and tritium isotopes for tracing landfill contamination in groundwater. Journal of Geochemical Exploration 172: 101-108. doi:10.1016/j. gexplo.2016.10.011.

Niswonger R.G., Panday S., Ibaraki M. (2011) MODFLOW-NWT, A Newton formulation for MODFLOW-2005. U.S. Geological Survey Techniques and Methods 6-A37.

Pollock D.W. (2016). User guide for MODPATH Version 7. A particle-tracking model for MODFLOW. U.S. Geological Survey OpenFile Report 2016-1086. doi:10.3133/ofr20161086.

Preziosi E., Frollini E., Zoppini A., Ghergo S., Melita M., Parrone D., Rossi D., Amalfitano S. (2019) Disentangling natural and anthropogenic impacts on groundwater by hydrogeochemical, isotopic and microbiological data: Hints from a municipal solid waste landfill. Waste Management 84: 245-255. doi:10.1016/j. wasman.2018.12.005

Rotiroti M., Fumagalli M., Bonomi T. (2014). How to manage potential groundwater contaminations by $\mathrm{As}, \mathrm{Fe}$ and $\mathrm{Mn}$ in lower Po Plain: a proposal from the case study of Cremona. Acque Sotterranee - Italian Journal of Groundwater 3(2): 9-16. doi:10.7343/ AS-070-14-0096

Stefania G.A., Zanotti C., Bonomi T., Fumagalli L., Rotiroti M. (2018) Determination of trigger levels for groundwater quality in landfills located in historically human-impacted areas. Waste Management 75: 400-406. doi:10.1016/j.wasman.2018.01.043

Stefania G.A., Rotiroti M., Buerge I.J., Zanotti C., Nava V., Leoni B., Fumagalli L., Bonomi T. (2019) Identification of groundwater pollution sources in a landfill site using artificial sweeteners, multivariate analysis and transport modeling. Waste Management 95: 116-128. doi:10.1016/j.wasman.2019.06.010

Tukey W. (1977). Exploratory Data Analysis. Addison-Wesley.

Vercesi P. (2009) Assetto idrogelogico della zona circostante la discarica di RSU di Vizzolo-Predabissi "Hydrogeology of the area surrounding the MSW landfill of Vizzolo-Predabissi”. Tecnical Report, Vizzolo Ambiente S.r.1., pp.86.

Ward, J.H., 1963. Hierarchical Grouping to Optimize an Objective Function. J. Am. Statistical Assoc. 58 (301), 236-244. https://doi org/10.1080/01621459.1963. 10500845. 